\title{
Pathophysiology and management of primary immune thrombocytopenia
}

\author{
Hirokazu Kashiwagi $\cdot$ Yoshiaki Tomiyama
}

Received: 29 March 2013/Revised: 7 May 2013/Accepted: 13 May 2013/Published online: 24 May 2013

(c) The Japanese Society of Hematology 2013

\begin{abstract}
Primary immune thrombocytopenia, or idiopathic thrombocytopenic purpura (ITP), is an autoimmune disorder characterized by isolated thrombocytopenia due to accelerated platelet destruction and impaired platelet production. Autoantibodies against platelet surface glycoproteins, such as GPIIb/IIIa and GPIb/IX complexes, play major roles in both platelet destruction and impaired platelet production, although autoantibody-independent mechanisms, such as $\mathrm{T}$ cell-mediated cytotoxicity, may also be involved in its pathogenesis. Recent advances in the localization of autoantigenic epitopes and the characterization of $\mathrm{T}$ cell functional abnormalities in ITP patients have improved our understanding of the pathophysiology of this disease. Although corticosteroids and splenectomy remain central to the treatment of ITP, a new class of drugs, i.e., thrombopoietin receptor agonists (TPO-RAs) and rituximab, have substantially broadened the therapeutic options for refractory ITP patients. Moreover, the success of TPO-RAs in ITP patients shows that reduced platelet production caused by impaired megakaryocytopoiesis plays a greater role in ITP than previously recognized.
\end{abstract}

Keywords Immune thrombocytopenia - Autoantibody Epitope $\cdot$ Thrombopoietin receptor agonist $\cdot$ Rituximab

H. Kashiwagi $(\square) \cdot$ Y. Tomiyama

Department of Hematology and Oncology, Osaka University

Graduate School of Medicine, 2-2 Yamadaoka, Suita,

Osaka 565-0871, Japan

e-mail: kashi@hp-blood.med.osaka-u.ac.jp

Y. Tomiyama

Department of Blood Transfusion, Osaka University Hospital,

Suita, Japan

\section{Introduction}

Primary immune thrombocytopenia (also known as idiopathic thrombocytopenic purpura; ITP) is an autoimmune disorder characterized by isolated thrombocytopenia without abnormalities in the erythroid and myeloid/lymphoid lineages [1-3]. The incidence of ITP in adults is estimated at approximately $1.6-3.9$ per 100,000 personyears [4-7]. It is conventionally thought that thrombocytopenia in ITP is caused by increased destruction of platelets opsonized by anti-platelet autoantibodies [8]. Abnormalities in megakaryocytopoiesis and impaired platelet production have also been suggested as etiologic factors $[9,10]$. The remarkable success of thrombopoietin receptor agonists (TPO-RAs) for ITP patients has demonstrated that impaired platelet production plays a substantial role in ITP. Selective B cell depletion with rituximab has also been shown to be effective in the treatment of ITP. In this review, we summarize recent advances in understanding of the pathophysiology of ITP, including its autoantigenic epitopes, and the current management of primary ITP.

\section{Pathophysiology of primary ITP}

Total platelet mass in the body is regulated by the balance between production and clearance of platelets. In hypoplastic thrombocytopenias, such as aplastic anemia or chemotherapy-induced thrombocytopenia, platelet counts are decreased due to reduced platelet production. In ITP, platelet mass shrinks as a result of accelerated platelet clearance, which is mainly due to autoantibody-mediated destruction by macrophages in spleen, and moderately impaired platelet production due to antibody- and/or 
cytotoxic $\mathrm{T}$ cell-mediated megakaryocytic damage (Fig. 1).

Abnormalities in B cells: production of anti-platelet autoantibodies

Historical experiments performed by Harrington and Hollingsworth in 1950 and subsequent studies, which demonstrated that the passive transfer of plasma [including immunoglobulin (Ig) G-rich fractions] from ITP patients induced the development of transient thrombocytopenia in healthy recipients, confirmed the immunologic etiology of this disease $[2,8,11,12]$. Subsequent studies in 1970s and 80 s revealed the plasma factors in question to be $\operatorname{IgG}$ antibodies against platelet surface glycoproteins, mainly GPIIb/IIIa and/or GPIb/IX [2]. Although plasma autoantibodies are clinically relevant, anti-platelet autoantibodies are more frequently detected in the platelet-associated (PA) form than in plasma form. The levels of PA autoantibodies, but not those of plasma autoantibodies, are correlated with the clinical course of ITP, and we and others have further shown that sera in ITP may contain antibodies against the cytoplasmic domain of GPIIIa and/or cytoplasmic proteins, such as vinculin, likely as a secondary effect of platelet destruction. Thus, in many cases, pathophysiologically important autoantibodies appear to be already bound to platelets $[2,13]$. PA anti-GPIIb/IIIa and anti-GPIb/IX antibodies are detected in $43-57 \%$ and $18-50 \%$ in chronic ITP (cITP) patients, respectively [13, 14].

For more than two decades, efforts have been focused on identifying target epitopes for PA autoantibodies. We and others reported that PA anti-GPIIb/IIIa antibodies frequently bind to cation-dependent conformational antigens, but not to another $\beta 3$ integrin, $\alpha v \beta 3[2,13]$. We also demonstrated that in one-third ITP patients (11 out of 34 patients) positive for PA anti-GPIIb/IIIa antibodies, reactivity of PA anti-GPIIb/IIIa antibodies is markedly impaired with KO-variant GPIIb/IIIa (a loss-of-function mutation in the $\beta$-propeller domain of GPIIb) as compared with wild-type GPIIb/IIIa [15]. These data suggest that the $\beta$-propeller domain of GPIIb is a hot spot for autoantigenic determinants. To further clarify the epitopes, we recently performed subsequent systematic analysis of the epitopes of PA anti-GPIIb/IIIa antibodies using human-mouse chimera GPIIb/IIIa constructs, as we found that most PA antiGPIIb/IIIa antibodies barely bind to mouse GPIIb/IIIa [16]. We detected PA anti-GPIIb/IIIa antibodies in 26 (34\%) of 76 ITP platelet eluates (PA autoantibodies eluted from washed ITP platelets by diethyl ether). We confirmed that autoantigenic epitopes are mainly localized on GPIIb, and further analyzed localization of autoantigens for 15 ITP eluates by employing various human-mouse chimera GPIIb complexed with human GPIIIa. The autoantigenic epitopes localized mainly on the $\mathrm{N}$-terminal half of the $\beta$-propeller

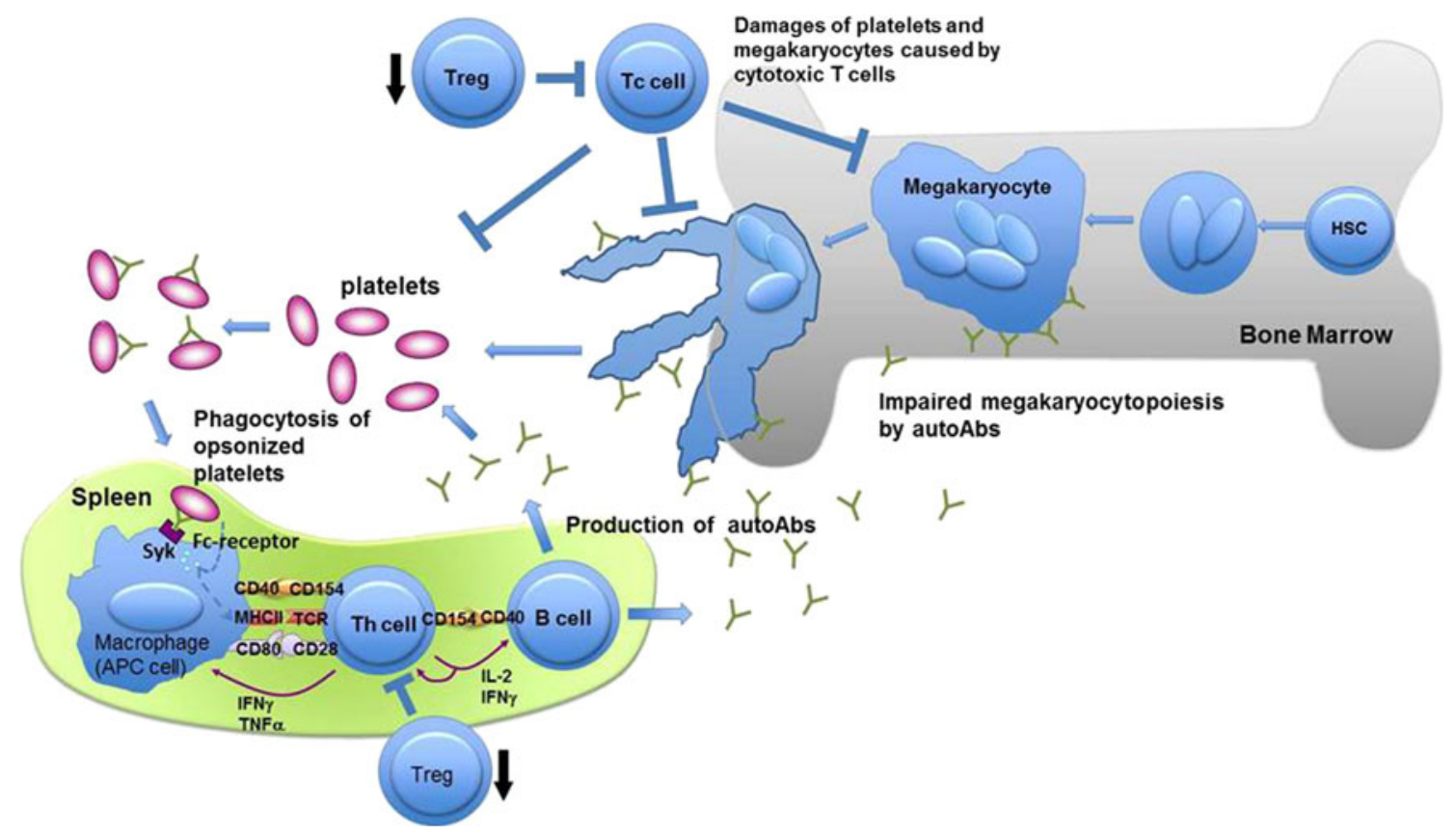

Fig. 1 Schematic representation of pathophysiology of cITP. Opsonized platelets by autoantibodies are destroyed by macrophages in spleen and peptide fragments expressed with MHC class II stimulate helper T cells, following activation of autoreactive B cells. Impaired Tregs fail to suppress this vicious cycle. Autoantibodies also suppress megakaryocytopoiesis. Autoreactive cytotoxic $\mathrm{T}$ cells may play a role in the destruction of platelets and megakaryocytes. Thrombopoietin receptor (TPO-R) agonists stimulate megakaryocyte proliferation and maturation. Rituximab targets CD20-positive B cells 
domain of GPIIb in all 15 patients examined (from the $\mathrm{N}$-terminus to W4:4-1 loop of the $\beta$-propeller domain; L1W235 of GPIIb) (Fig. 2). We further identified three main autoantigenic recognition sites within the region $(\mathrm{a}-\mathrm{c}$ regions in Fig. 3). Interestingly, at least in three ITP patients the reactivity of autoantibodies was completely destroyed by only one amino acid substitution (R139G for two patients, and $\mathrm{G} 44 \mathrm{~N}$ for one patient), suggesting that the target epitopes were localized on very restricted region on GPIIb in these three patients [16]. Regarding the epitopes of anti-GPIb/IX autoantibodies, He et al. [17] examined their localization by employing short linear fragments of GPIb $\alpha$. They showed that six of 16 anti-GPIb/ IX antibodies in ITP patients recognize a short amino acid sequence (amino acids 333-341) in GPIb $\alpha$. Again, the

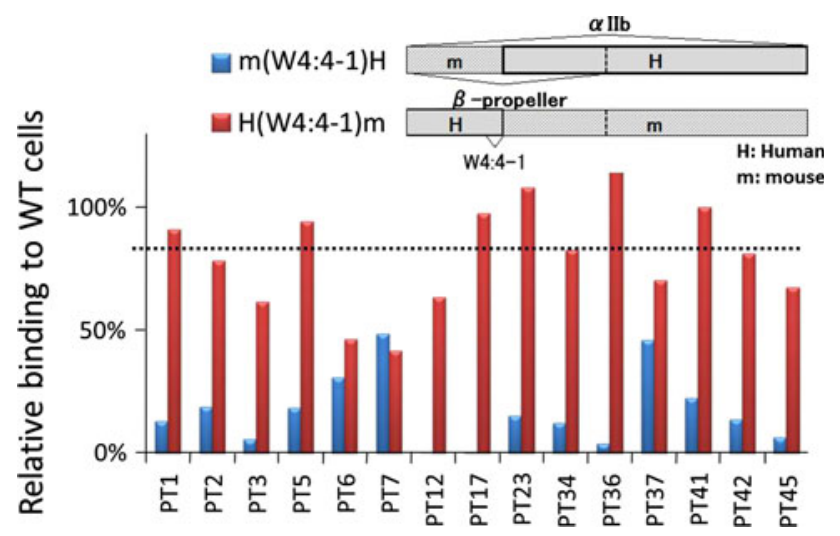

Fig. 2 Binding of platelet-associated (PA) anti-GPIIb/IIIa antibodies in ITP patients to human-mouse chimera GPIIb/IIIa. Relative bindings of PA antibodies to 293T cells that expressed human GPIIb replaced the $\mathrm{N}$-terminal half of the $\beta$-propeller domain with mouse [m(W4:4-1)H, blue] and 293T cells expressing mouse GPIIb replaced the N-terminal half of the $\beta$-propeller domain with human [H(W4:41)m, red] are shown autoantigenic epitopes for anti-GPIb-IX antibodies seem to be localized on the limited region.

A genetic analysis of the Fab region of immunoglobulin (Ig) using phage display libraries constructed from splenocytes from cITP patients demonstrated that antiplatelet autoantibodies in cITP use highly restricted Ig variable regions [18]. We and others have also demonstrated that many of PA anti-GPIIb/IIIa antibodies show restricted $\kappa / \lambda$-chain usage $[16,19]$. These data suggest that the antigenic repertoire in ITP is fairly limited and that anti-platelet antibodies are produced in limited B cell clones in many ITP patients. In HIV-, HCV- and Helicobacter pylori (H. pylori)-associated secondary ITP, cross reactivity of platelet antibodies with these pathogens has been reported [20-22]. By analogy to these secondary ITP, molecular mimicry of unknown pathogens with limited regions of platelet glycoproteins may trigger expansion of auto-reactive B cell clones in primary ITP, and autoantibody production from the $\mathrm{B}$ cell clones may be affected by $\mathrm{T}$ cell abnormalities, as described in the next section (Fig. 1).

\section{Abnormalities in $\mathrm{T}$ cells}

Recently, several lines of evidences have linked $\mathrm{T}$ cell abnormalities to the pathogenesis of ITP. Kuwana et al. [23] demonstrated the presence of auto-reactive $T$ cell clones against cryptic GPIIb/IIIa epitopes in cITP patients. Other studies have shown that cITP patients exhibit an imbalanced Th1/Th2 ratio [24], increase of Th17 cells and IL17 levels [25], increase of oligoclonal T cells [26], and the presence of cytotoxic $\mathrm{T}$ cells against autologous platelets [27].

The functions of regulatory $\mathrm{T}$ cells (Treg) in ITP have been extensively examined, as emergence of anti-platelet
Fig. 3 Demonstration of three major recognition sites $(A, B$ and $C$ ) of PA anti-GPIIb/IIIa autoantibodies in ITP patients. Shown is a crystal structure of the resting form of GPIIb/IIIa with two different angles made by PyMOL Version 1.4 software (DeLano Scientific LLC). The N-terminal half and the whole region of the $\beta$ propeller domain of GPIIb are indicated in yellow and by the dotted circle, respectively. Each loop essential for PA antibody bindings is distinctly colored

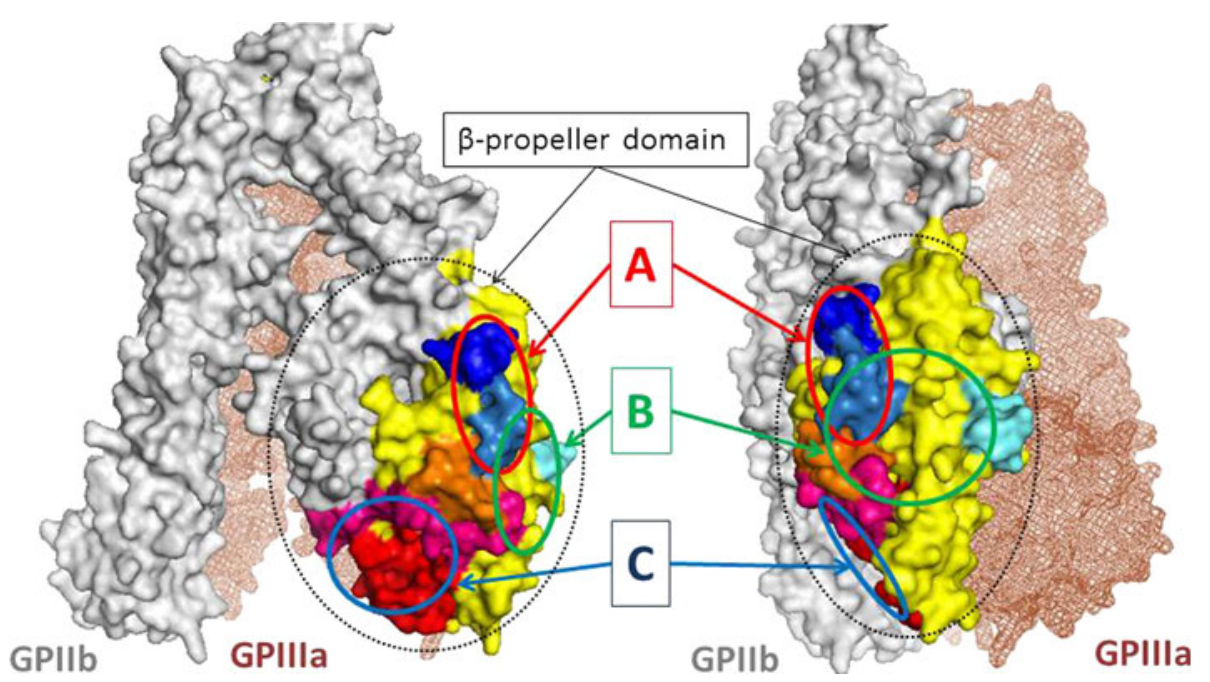


autoantibodies and anti-platelet cytotoxic $\mathrm{T}$ cells is an expected consequence of the loss of immunological tolerance to self-antigens. Tregs are $\mathrm{T}$ cells marked by $\mathrm{CD} 4{ }^{+} \mathrm{CD} 25^{+} \mathrm{Foxp}^{+}$, which account for $5-10 \%$ of the peripheral $\mathrm{CD}^{+}{ }^{+} \mathrm{T}$ cell population and play essential roles in self-tolerance by suppression of cell- and antibodymediated immune responses [28]. Several reports demonstrated reduction in Treg numbers and/or impairment of Treg function in ITP patients [29, 30]. Very recently, Nishimoto et al. [31] demonstrated that approximately $40 \%$ of Treg-deficient mice became thrombocytopenic, which lasted for up to 5 weeks. The thrombocytopenic mice displayed IgG anti-platelet antibodies, which predominantly react to GPIb/IX, and transfer of purified Tregs into the mice prevented thrombocytopenia [31, 32]. Stasi et al. [33] demonstrated that a reduced number and a defective suppressive capacity of Tregs in ITP patients were restored in responders of rituximab, suggesting abnormalities in Tregs in ITP patients may be regulated by interaction between $\mathrm{T}$ and $\mathrm{B}$ cells.

\section{Mechanism of accelerated platelet clearance}

Opsonized platelets by anti-platelet IgG antibodies are destructed mainly by macrophages in spleen via lowaffinity Fc receptors, Fc $\gamma$ RIIA and Fc $\gamma$ RIIIA [34]. Fc $\gamma$ RIIA and Fc $\gamma$ RIIIA have immunoreceptor tyrosine-based activation motif (ITAM) in the cytoplasmic regions, and phosphorylation of ITAM domain induced by Fc binding, followed by Syk phosphorylation, activates phagocytosis [35]. Several reports have also suggested the involvement of another inhibitory Fc $\gamma \mathrm{R}, \mathrm{Fc} \gamma \mathrm{RIIB}$, in the control of phagocytosis. Fc $\gamma$ RIIB contains an immunoreceptor tyrosine-based inhibitory motif (ITIM) and inhibits phagocytosis and pro-inflammatory cytokine release by monocytes/ macrophages and dendritic cells. Samuelsson et al. [36] showed that Fc $\gamma$ RIIB on splenic macrophages is required for the protective effects of intravenous immunoglobulin against platelet consumption in a murine ITP model. Sifting of the monocyte Fc $\gamma \mathrm{R}$ balance toward the inhibitory Fc $\gamma$ RIIB has been also demonstrated in ITP patients responded to $H$. pylori eradication [37] or high-dose dexamethasone treatment [38]. In addition to $\mathrm{Fc}$-mediated phagocytosis, platelet autoantibodies may fix complements, enhancing opsonization or facilitating direct platelet lysis [39-41]. Moreover, Nardi et al. [20] showed that antiGPIIIa antibodies found in an HIV-associated ITP patient caused platelet destruction via induction of reactive oxygen species (ROS), and that these mechanisms may also contribute to primary ITP. Some patients with ITP lack detectable anti-platelet autoantibodies, and platelet clearance in these cases may result from $\mathrm{CD} 8^{+} \mathrm{T}$ cell-mediated cytotoxicity $[27,42]$.
Impaired platelet production

Impairment of platelet production in ITP patients has been demonstrated directly or indirectly by morphological studies of megakaryocytes, megakaryocytic colony formation and platelet kinetic studies.

\section{Abnormalities in morphology of megakaryocytes}

Morphological abnormalities in megakaryocytes of cITP patients, e.g. increase of immature megakaryocytes, disappearance of platelet production, and degenerative change of nucleus and cytoplasm, have been observed by optical microscopic analysis since the 1940s [2]. Ultrastructural analysis of megakaryocytes of 11 ITP patients showed extensive apoptotic and para-apoptotic changes, such as dilation of endoplasmic reticulum, swollen mitochondria, dilation of the demarcation membrane system, nuclear fragmentation and chromatin condensation, in about $80 \%$ of ITP megakaryocytes [43]. These (para-)apoptotic changes can be induced in megakaryocytes obtained by suspension culture of $\mathrm{CD} 34^{+}$cells with ITP plasma [43].

\section{Suppression of megakaryocytopoiesis}

Since the expression levels of GPIb/IX and GPIIb/IIIa increases during the maturation process of megakaryocytes [44], it is very plausible that anti-platelet autoantibodies against GPIb/IX and/or GPIIb/IIIa attack megakaryocytes as well as platelets. Two in vitro studies using megakaryocyte culture systems from cord blood-derived CD $34^{+}$ cells showed that autoantibodies against anti-GPIb/IX and GPIIb/IIIa in ITP patients reduce megakaryocyte production and maturation $[45,46]$. Antibodies bound to megakaryocytes may induce apoptosis via ROS production and/ or activation of the PI3 K/Akt pathway, in addition to antibody-dependent cellular toxicity and complementdependent cytotoxicity [47]. In contrast, Yang et al. [48] demonstrated that most ITP plasma boosts megakaryocyte quantity but impairs megakaryocyte maturation, resulting in significantly less polyploidy cells and platelet release, and that cell apoptosis is inhibited in these immature megakaryocytes. Megakaryocytes in ITP patients may also be attacked by cytotoxic T cells in bone marrow [49].

\section{Platelet kinetic studies and serum (plasma) thrombopoietin concentration}

Intensive studies of platelet kinetics using ${ }^{111}$ indiumlabeled autologous platelet transfusion were performed in 1980s, and showed that platelet production remains normal or is decreased in two-thirds of cITP patients compared with normal subjects $[9,50]$, suggesting that accelerated 
platelet clearance may not be the sole etiologic factor underlying thrombocytopenia in ITP. We assessed platelet turnover and production using reticulated platelets, which are RNA-rich young platelets stained with thiazole orange, by flow cytometry (FCM) [51]. Percentage (RP \%) and absolute number (RP number) of reticulated platelets represent turnover and production of platelets, respectively. In cITP patients, RP \% was markedly increased compared with healthy control (healthy control $7.7 \pm 2.7 \%$, cITP $23.8 \pm 11.6, \quad P<0.001)$, whereas $\mathrm{RP}$ number was decreased (control $17.0 \pm 6.6 \times 10^{3} / \mu \mathrm{l}$, cITP $8.3 \pm 4.6$, $P<0.05)$. These data confirm that both rapid platelet turnover and impaired platelet production occur in ITP patients. Two methods are available for measuring RNA-rich platelets: the RP \% method using FCM and the IPF \% method (immature platelet fraction) performed using the Sysmex XE-2100 (or XE-5000) autoanalyzer. We have compared these two methods in parallel for their utility in differential diagnosis between ITP and AA. The sensitivity ( $82 \%)$ and specificity $(93 \%)$ of the RP \% method for the diagnosis of ITP were much better than those of the IPF $\%$ method (sensitivity $67 \%$, specificity $63 \%$ ). Although IPF \% is measured automatically and easy to use, we should take its moderate sensitivity and specificity into account [52].

TPO is the major physiologic regulator of platelet production, and it is continuously synthesized and secreted predominantly in the liver, with no translational or posttranslational regulation [53, 54]. Serum (plasma) TPO level is regulated by consumption of TPO by its binding to the TPO receptor (c-Mpl) on platelets or megakaryocytes.
When platelet mass increases, the relative amount of TPO to megakaryocytes decreases, leading to suppression of platelet production. Conversely, when platelet mass is reduced, the relative amount of TPO to megakaryocytes rises. These mechanisms, referred to collectively as "the sponge theory", contribute to homeostasis of platelet number. In fact, TPO levels of patients with aplastic anemia or chemotherapy-induced thrombocytopenia are markedly elevated. In contrast, TPO levels are not elevated, or only slightly elevated, in patients with ITP [51, 55]. Taken together with the moderately impaired platelet production in ITP, relatively low levels of TPO indicate that ITP may represent a good candidate for treatment with TPO-RAs.

\section{Current management of ITP}

Differences in the rates of platelet count increase following $H$. pylori eradication in ITP have been reported for different regions. In Japan, H. pylori screening and eradication appear to be warranted, given the high background prevalence of $H$. pylori infection and the high platelet response rate to eradication, whereas in the United States and Europe its value is uncertain [56]. Such inter-ethnic differences should be considered when developing treatment guidelines for chronic ITP. In this context, a reference guide for management of adult cITP was published in 2012 by the study group of the Specific Disease Treatment Research Program for Intractable Diseases of the Ministry

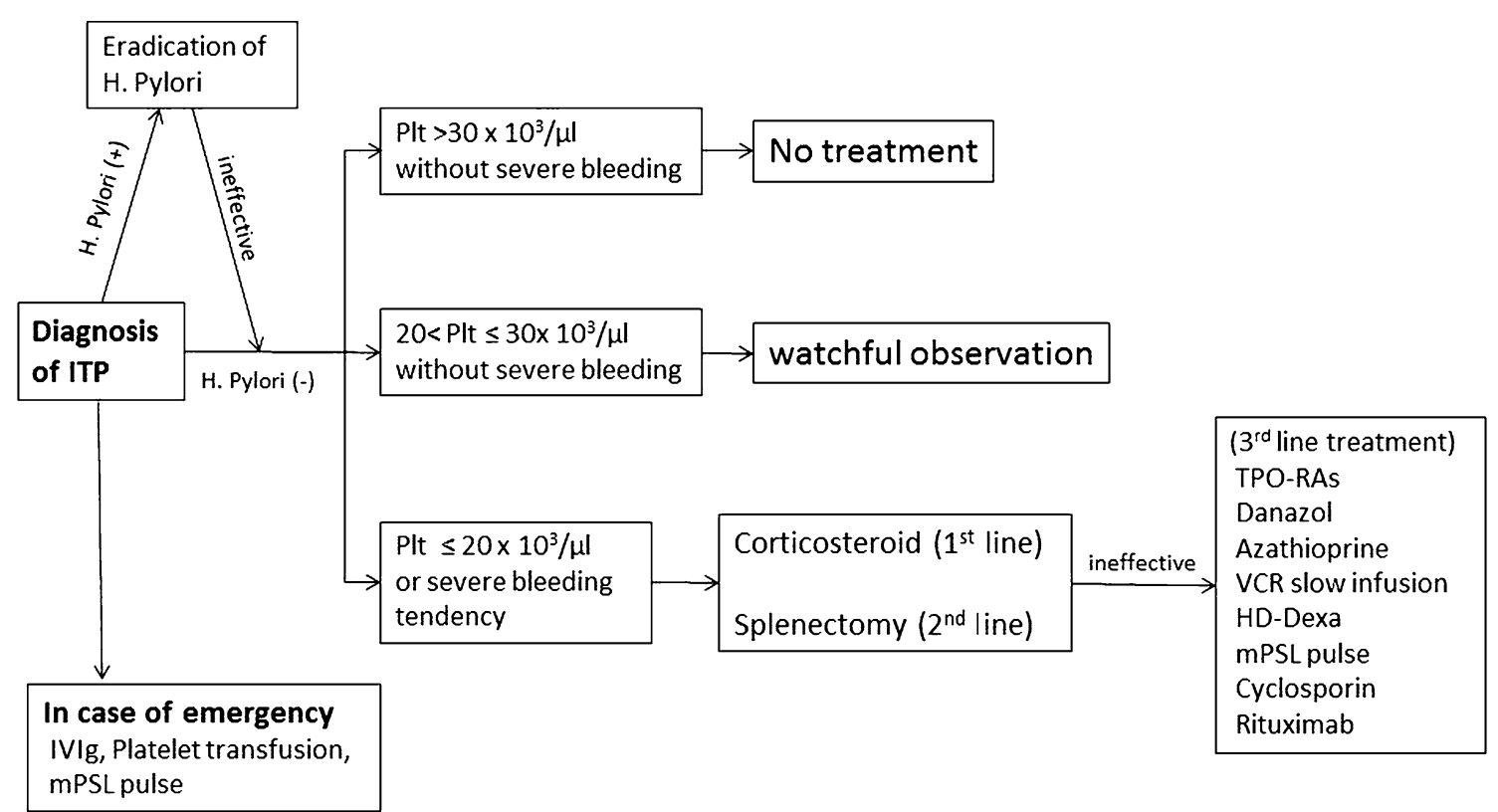

Fig. 4 The 2012 reference guide for management of adult cITP published from the study group of the Specific Disease Treatment Research Program for Intractable Diseases of the Ministry of Health
Labour and Welfare of Japan. IVIg intravenous immunoglobulin, mPSL methylprednisolone, VCR vincristine, HD-Dexa high-dose dexamethasone 
of Health, Labour, and Welfare in Japan (Fig. 4). The reference guide corresponds in large part with a recently published international consensus report [3], except eradication of H. pylori. When H. pylori is negative or platelet response to the eradication is not obtained, these guidelines recommend starting treatment if platelet counts fall below $20-30 \times 10^{3} / \mu \mathrm{L}$, and/or severe bleeding is observed. First line treatment is corticosteroids, usually prednisolone, although complete remission, which is defined as more than $100 \times 10^{3} / \mu \mathrm{l}$ without medication, would be achieved in only $\sim 20 \%$ of patients. Splenectomy is recommended for corticosteroid-resistant patients as a second-line treatment. Splenectomy has a 60-year record of success for achieving durable remissions in approximately two-thirds of patients [57]. For refractory ITP patients in whom corticosteroids and splenectomy fail, many drugs have been tried as third-line treatments, including TPO-RAs and rituximab, although only TPO-RAs are approved for the treatment of ITP in Japan.

\section{TPO-RAs}

Recombinant human TPO (rhTPO) and pegylated recombinant human megakaryocyte growth and development factor (PEG-rHuMGDF) were developed for clinical studies in thrombocytopenic disorders [53]. PEG-rHuMGDF is a truncated form of TPO that contains the first 163 amino acids of endogenous TPO. However, PEGrHuMGDF paradoxically induced persistent thrombocytopenia in 13 of 325 healthy volunteers. This thrombocytopenia was caused by an antibody to PEG-rHuMGDF that cross-reacted with endogenous TPO and neutralized its biological activity [58]. Although rhTPO did not show such adverse effects, the development of both rhTPO and PEGrHuMGDF was stopped in 1998. Subsequently, efforts to produce safer TPO-related products continued, and we are now able to use two TPO-RAs for refractory ITP patients in the clinical setting $[59,60]$.

\section{Eltrombopag}

Eltrombopag is an orally available small non-peptide molecule with a molecular weight of $546 \mathrm{Da}$. It was identified by screening of large libraries of small nonpeptide molecules for the ability to stimulate STATs in TPO-dependent cell lines [61]. Eltrombopag binds to the transmembrane region of the TPO receptor, and activates JAK/STAT and RAS/RAF/MAPK pathways [59].

A double-blind, placebo-control study in adults with ITP patients with platelet counts of lower than $30,000 / \mu \mathrm{L}$ (RAISE study) showed $\sim 80 \%$ patients in the eltrombopag group responded to treatment at least once during the study, compared with $28 \%$ patients in the placebo group
[62]. Importantly, similar responses were obtained irrespective of splenectomy status and $59 \%$ of patients in the eltrombopag group could reduce concomitant treatment (mostly corticosteroids). Three ( $2 \%$ ) out of 135 patients receiving eltrombopag had thromboembolic events [2 pulmonary embolism (PE) and 1 deep-vein thrombosis (DVT)]. Nine (7\%) and five (4\%) eltrombopag-treated patients showed mild increases in alanine aminotransferase and total bilirubin, respectively. A recently published longterm study (median 100 weeks) of eltrombopag (EXTEND study) showed maintenance of high efficiency (254/299, $85 \%)$ of eltrombopag in long-term use [63]. Twenty-one thromboembolic events [nine deep-vein thrombosis (DVT), five central nervous system ischemic events, four myocardial infarction, and three pulmonary embolisms (PE)] were reported in sixteen $(16 \%)$ patients and two patients were diagnosed with lymphoma.

Patient ethnicity may affect the pharmacokinetics of eltrombopag. AUC exposure to eltrombopag was approximately twofold greater among Japanese healthy volunteers than among non-Asian (predominantly Caucasian) volunteers, and $87 \%$ greater among ITP patients of East Asian descent compared to non-East Asian ITP patients [64, 65]. Multiple factors, including body weight differences and genetic differences in metabolizing enzymes such as UGT1A1, UGT1A3, CYP1A2, and CYP2C8 and transporters, may contribute to the differences between Japanese and Caucasian patients [64]. From these data, it has been recommended that for patients of East Asian ancestry (such as Japanese, Korean, Chinese, and Taiwanese) the initial dose of eltrombopag should be reduced to $25 \mathrm{mg}$ once daily. Notably, a recent Japanese clinical trial evaluated the efficacy and safety of eltrombopag at a starting dose of $12.5 \mathrm{mg}$ and a maximum dose of $50 \mathrm{mg}$ in the treatment of Japanese patients with previously treated chronic ITP [66]. During the first 3 weeks treated with $12.5 \mathrm{mg}$ eltrombopag, $22 \%(5 / 23)$ of Japanese patients responded. Since the disease state is chronic in nature and only $12.5 \mathrm{mg}$ tablets are available in Japan to date, it is possible that $25 \mathrm{mg}$ every-other-day administration as a starting dose may be suitable for some chronic ITP patients of East Asian ancestry to prevent overshooting of platelet count.

\section{Romiplostim}

Romiplostim is a rationally designed "peptibody" composed of an $\mathrm{Fc}$ fragment with two identical peptide sequences linked via polyglycine covalently bound at residue 228 of the heavy chain [59]. Its half-life is substantially lengthened by the $\mathrm{Fc}$ fragments, and it is administered subcutaneously once a week. Romiplostim competitively binds to the TPO receptor with intrinsic TPO, but the receptor-binding peptide component shows 
no sequence homology with human TPO and there have been no reports of the production of clinically significant antibodies against romiplostim.

A short-term (24 weeks), double-blind randomized controlled trial in adult cITP patients demonstrated $88 \%$ (36/41) and $79 \%$ (33/42) overall platelet response rates in non-splenectomised and splenectomised patients given romiplostim, respectively [67]. A 52-week study in 234 non-splenectomised adult cITP patients, in which randomly assigned romiplostim or standard of care (SOC), showed a higher rate of a platelet response and lower incidence of treatment failure in the romiplostim group [68]. It has been reported that cITP patients experience poor quality-of-life (QOL) [69], and this study showed that the romiplostim group had significantly greater improvement of QOL compared with the SOC group at 52 weeks [68]. Thromboembolic events were reported in $4 \%(6 / 154)$ in the romiplostim group and $3 \%(2 / 75)$ in the SOC group, with no statistically significant differences, and no hematologic malignancy was reported in either group. An open-label extension study of romiplostim for up to 3.5 years in 44 Japanese patients also demonstrated very high response rate $(96 \%)$ [70]. The median dose of romiplostim was similar compared with the studies of other ethnic origins ( $3.8 \mu \mathrm{g} / \mathrm{kg}$ in the Japanese study, $4.0 \mu \mathrm{g} / \mathrm{kg}$ in the other). The only thromboembolic event was transient ischemic attack in a patient who had a history of paroxysmal atrial fibrillation [70].

\section{Rituximab}

Rituximab is a chimeric monoclonal antibody that specifically depletes B cells from the blood, lymph nodes, and bone marrow by targeting CD20, which is expressed on the surface of premature and mature B cells [71]. Rituximab has been widely used for the treatment of B cell malignancies, such as indolent and aggressive non-Hodgkin lymphoma, and chronic lymphocytic leukemia, and its effectiveness have been well-established [72, 73]. In addition, rituximab has been used off-label for autoimmune diseases, especially for ITP patients in Europe and the United States [74]. Recent reviews have suggested that the usual rituximab treatment $\left(375 \mathrm{mg} / \mathrm{m}^{2}\right.$ weekly $\left.\times 4\right)$ is effective in around $40-60 \%$ cITP patients, and that the response in more than $50 \%$ of responders lasts at least 1 year, although the 5 -year response rate was as low as $20 \%$ in adults $[75,76]$. Some reports have shown that smaller doses of rituximab $(100 \mathrm{mg}$ weekly $\times 4)$ have almost the same effects as the usual dose [77, 78]. One report showed that retreatment with standard dose rituximab was successful in $\sim 75 \%$ of patients who had responded to their initial course of rituximab [79]. Evidence-based ASH 2011 guidelines suggest that rituximab may be considered for patients at risk of bleeding who have failed one line of therapy such as corticosteroids, intravenous immunoglobulin or splenectomy [80]. Overall, the safety profile of the rituximab therapy is good. However, the possible occurrence of life-threatening infectious complications, such as hepatitis B virus reactivation and progressive multifocal leukoencephalopathy, should be noted, especially in countries, such as Japan, with high incidence of HBV carriage.

The mechanism of action of rituximab in ITP may not be as straightforward as it first appears [74]. In most studies published to date, there were two patterns of response; the majority of responders responded to rituximab within 4 weeks, whereas, the rest responded more slowly, several weeks or even months after rituximab treatment. The response in early responders to rituximab is too rapid to be explained by the depletion of autoantibodies. Instead, it has been proposed that opsonized B cells with rituximab block macrophage $\mathrm{Fc}$-receptor function, reducing platelets destruction in the spleen $[81,82]$. Very recently, Audia et al. [83] reported that rituximab-induced total B cell depletion may not be correlated with its therapeutic effects. In this context, Stasi et al. [26, 33] demonstrated that the therapeutic efficacy of rituximab may be due to normalization of the abnormal autoreactive $\mathrm{T}$ cell responses and Treg function in ITP patients. A combination of these mechanisms likely plays a role in the efficacy of rituximab in ITP.

\section{Conclusion}

It is now clear that ITP is caused by both increased platelet clearance and decreased platelet production. However, the underlying abnormalities involved in the development of ITP appear to be complex and mostly remain to be determined. Recent progress of ITP treatment enables us to use three distinct approaches to increase platelet mass: (1) by suppression of platelet clearance, e.g. splenectomy, (2) by suppression or modification of abnormal immune responses, e.g. corticosteroids or rituximab, and (3) by stimulation of platelet production, e.g. TPO-RAs. Further clinical studies are needed to establish the best strategies using these approaches for the treatment of ITP patients, together with the development of new treatments aimed at curing ITP through elucidation of its pathophysiology.

Acknowledgments This work was supported by Grant-in Aid for Scientific Research from the Ministry of Education, Culture, Sports, Science and Technology in Japan and the Ministry of Health, Labour and Welfare in Japan.

\section{References}

1. Cines DB, Blanchette VS. Immune thrombocytopenic purpura. N Engl J Med. 2002;346:995-1008. 
2. McMillan R. The pathogenesis of chronic immune thrombocytopenic purpura. Semin Hematol. 2007;44(Suppl 5):S3-11.

3. Provan D, Stasi R, Newland AC, Blanchette VS, Bolton-Maggs $\mathrm{P}$, Bussel JB, et al. International consensus report on the investigation and management of primary immune thrombocytopenia. Blood. 2010;115:168-86.

4. Frederiksen H, Schmidt K. The incidence of idiopathic thrombocytopenic purpura in adults increases with age. Blood. 1999; 94:909-13.

5. Neylon AJ, Saunders PWG, Howard MR, Proctor SJ, Taylor PRA. Clinically significant newly presenting autoimmune thrombocytopenic purpura in adults: a prospective study of a population-based cohort of 245 patients. Br J Haematol. 2003; 122:966-74.

6. Schoonen WM, Kucera G, Coalson J, Li L, Rutstein M, Mowat F, et al. Epidemiology of immune thrombocytopenic purpura in the general practice research database. Br J Haematol. 2009;145: 235-44.

7. Kurata Y, Fujimura K, Kuwana M, Tomiyama Y, Murata M. Epidemiology of primary immune thrombocytopenia in children and adults in Japan: a population-based study and literature review. Int J Hematol. 2011;93:329-35.

8. Harrington WJ, Minnich V, Hollingsworth JW, Moore CV. Demonstration of a thrombocytopenic factor in the blood of patients with thrombocytopenic purpura. J Lab Clin Med. 1951; 38:1-10.

9. Ballem PJ, Segal GM, Stratton JR, Gernsheimer T, Adamson JW, Slichter SJ. Mechanisms of thrombocytopenia in chronic autoimmune thrombocytopenic purpura. Evidence of both impaired platelet production and increased platelet clearance. J Clin Invest. 1987;80:33-40.

10. McMillan R, Nugent D. The effect of antiplatelet autoantibodies on megakaryocytopoiesis. Int J Hematol. 2005;81:94-9.

11. Shulman NR, Marder VJ, Weinrach RS. Similarities between known antiplatelet antibodies and the factor responsible for thrombocytopenia in idiopathic purpura. Physiologic, serologic and isotopic studies. Ann NY Acad Sci. 1965;124:499-542.

12. Schwartz RS. Immune thrombocytopenic purpura-from agony to agonist. N Engl J Med. 2007;357:2299-301.

13. Tomiyama Y, Kosugi S. Autoantigenic epitopes on platelet glycoproteins. Int J Hematol. 2005;81:100-5.

14. McMillan R, Wang L, Tani P. Prospective evaluation of the immunobead assay for the diagnosis of adult chronic immune thrombocytopenic purpura (ITP). J Thromb Haemost. 2003;1: 485-91.

15. Kosugi S, Tomiyama Y, Honda S, Kato H, Kiyoi T, Kashiwagi H, et al. Platelet-associated anti-GPIIb-IIIa autoantibodies in chronic immune thrombocytopenic purpura recognizing epitopes close to the ligand-binding site of glycoprotein (GP) IIb. Blood. 2001; 98:1819-27.

16. Kiyomizu K, Kashiwagi H, Nakazawa T, Tadokoro S, Honda S, Kanakura Y, et al. Recognition of highly restricted regions in the $\beta$-propeller domain of $\alpha \mathrm{IIb}$ by platelet-associated anti- $\alpha \mathrm{IIb} \beta 3$ autoantibodies in primary immune thrombocytopenia. Blood. 2012;120:1499-509.

17. He R, Reid DM, Jones CE, Shulman NR. Extracellular epitopes of platelet glycoprotein $\mathrm{Ib}$ alpha reactive with serum antibodies from patients with chronic idiopathic thrombocytopenic purpura. Blood. 1995;86:3789-96.

18. Roark JH, Bussel JB, Cines DB, Siegel DL. Genetic analysis of autoantibodies in idiopathic thrombocytopenic purpura reveals evidence of clonal expansion and somatic mutation. Blood. 2002;100:1388-98.

19. McMillan R, Lopez-Dee J, Bowditch R. Clonal restriction of platelet-associated anti-GPIIb/IIIa autoantibodies in patients with chronic ITP. Thromb Haemost. 2001;85:821-3.
20. Nardi M, Tomlinson S, Greco MA, Karpatkin S. Complementindependent, peroxide-induced antibody lysis of platelets in HIV1-related immune thrombocytopenia. Cell. 2001;106:551-61.

21. Zhang W, Nardi MA, Borkowsky W, Li Z, Karpatkin S. Role of molecular mimicry of hepatitis $\mathrm{C}$ virus protein with platelet GPIIIa in hepatitis C-related immunologic thrombocytopenia. Blood. 2009;113:4086-93.

22. Takahashi T, Yujiri T, Shinohara K, Inoue Y, Sato Y, Fujii Y, et al. Molecular mimicry by Helicobacter pylori CagA protein may be involved in the pathogenesis of $\mathrm{H}$. pylori-associated chronic idiopathic thrombocytopenic purpura. $\mathrm{Br} \mathrm{J}$ Haematol. 2004;124:91-6.

23. Kuwana M, Kaburaki J, Ikeda Y. Autoreactive T cells to platelet GPIIb-IIIa in immune thrombocytopenic purpura. Role in production of anti-platelet autoantibody. $\mathrm{J}$ Clin Invest. 1998;102:1393-402.

24. Panitsas FP, Theodoropoulou M, Kouraklis A, Karakantza M, Theodorou GL, Zoumbos NC, et al. Adult chronic idiopathic thrombocytopenic purpura (ITP) is the manifestation of a type-1 polarized immune response. Blood. 2004;103:2645-7.

25. Hu Y, Ma DX, Shan NN, Zhu YY, Liu XG, Zhang L, et al. Increased number of Tc17 and correlation with Th17 cells in patients with immune thrombocytopenia. PLoS ONE. 2011;6: e26522.

26. Stasi R, Del Poeta G, Stipa E, Evangelista ML, Trawinska MM, Cooper N, et al. Response to B-cell depleting therapy with rituximab reverts the abnormalities of T-cell subsets in patients with idiopathic thrombocytopenic purpura. Blood. 2007;110:2924-30.

27. Olsson B, Andersson PO, Jernås M, Jacobsson S, Carlsson B, Carlsson LM, et al. T-cell-mediated cytotoxicity toward platelets in chronic idiopathic thrombocytopenic purpura. Nat Med. 2003;9:1123-4.

28. Sakaguchi S. Naturally arising Foxp3-expressing CD25 + CD4 + regulatory T cells in immunological tolerance to self and non-self. Nat Immunol. 2005;6:345-52.

29. Sakakura M, Wada H, Tawara I, Nobori T, Sugiyama T, Sagawa $\mathrm{N}$, et al. Reduced $\mathrm{Cd} 4+\mathrm{Cd} 25+\mathrm{T}$ cells in patients with idiopathic thrombocytopenic purpura. Thromb Res. 2007;120: 187-93.

30. Yu J, Heck S, Patel V, Zhu XJ, Hou Y, Jun P, et al. Defective circulating CD25 regulatory $\mathrm{T}$ cells in patients with chronic immune thrombocytopenic purpura. Blood. 2008;112:1325-8.

31. Nishimoto T, Satoh T, Takeuchi T, Ikeda Y, Kuwana M. Critical role of $\mathrm{CD} 4(+) \mathrm{CD} 25(+)$ regulatory $\mathrm{T}$ cells in preventing murine autoantibody-mediated thrombocytopenia. Exp Hematol. 2012; 40:279-89.

32. Nishimoto T, Satoh T, Simpson EK, Ni H, Kuwana M. Predominant autoantibody response to GPIb/IX in a regulatory T-cell-deficient mouse model for immune thrombocytopenia. J Thromb Haemost. 2013;11:369-72.

33. Stasi R, Cooper N, Del Poeta G, Stipa E, Laura Evangelista M, Abruzzese E, et al. Analysis of regulatory T-cell changes in patients with idiopathic thrombocytopenic purpura receiving $\mathrm{B}$ cell-depleting therapy with rituximab. Blood. 2008;112:1147-50.

34. Stasi R. Pathophysiology and therapeutic options in primary immune thrombocytopenia. Blood Tranfus. 2011;9:262-73.

35. Podolanczuk A, Lazarus AH, Crow AR, Grossbard E, Bussel JB. Of mice and men: an open-label pilot study for treatment of immune thrombocytopenic purpura by an inhibitor of Syk. Blood. 2009;113:3154-60.

36. Samuelsson A, Towers TL, Ravetch JV. Anti-inflammatory activity of IVIG mediated through the inhibitory $\mathrm{Fc}$ receptor. Science. 2001;291:484-6.

37. Asahi A, Nishimoto T, Okazaki Y, Suzuki H, Masaoka T, Kawakami Y, et al. Helicobacter pylori eradication shifts monocyte Fc gamma receptor balance toward inhibitory Fc gamma RIIB in 
immune thrombocytopenic purpura patients. J Clin Invest. 2008; 118:2939-49.

38. Liu XG, Ma SH, Sun JZ, Ren J, Shi Y, Sun L, et al. High-dose dexamethasone shifts the balance of stimulatory and inhibitory Fc gamma receptors on monocytes in patients with primary immune thrombocytopenia. Blood. 2011;117:2061-9.

39. Tsubakio T, Tani P, Curd JG, McMillan R. Complement activation in vitro by antiplatelet antibodies in chronic immune thrombocytopenic purpura. Br J Haematol. 1986;63:293-300.

40. Peerschke EI, Andemariam B, Yin W, Bussel JB. Complement activation on platelets correlates with a decrease in circulating immature platelets in patients with immune thrombocytopenic purpura. Br J Haematol. 2010;148:638-45.

41. Najaoui A, Bakchoul T, Stoy J, Bein G, Rummel MJ, Santoso S, et al. Autoantibody-mediated complement activation on platelets is a common finding in patients with immune thrombocytopenic purpura (ITP). Eur J Haematol. 2012;88:167-74.

42. Chow L, Aslam R, Speck ER, Kim M, Cridland N, Webster ML, et al. A murine model of severe immune thrombocytopenia is induced by antibody- and CD8 $+\mathrm{T}$ cell-mediated responses that are differentially sensitive to therapy. Blood. 2010;115:1247-53.

43. Houwerzijl EJ, Blom NR, van der Want JJ, Esselink MT, Koornstra JJ, Smit JW, et al. Ultrastructural study shows morphologic features of apoptosis and para-apoptosis in megakaryocytes from patients with idiopathic thrombocytopenic purpura. Blood. 2004;103:500-6.

44. Lepage A, Leboeuf M, Cazenave JP, de la Salle C, Lanza F, Uzan G. The alpha(IIb)beta(3) integrin and GPIb-V-IX complex identify distinct stages in the maturation of CD34(+) cord blood cells to megakaryocytes. Blood. 2000;96:4169-77.

45. Chang M, Nakagawa PA, Williams SA, Schwartz MR, Imfeld KL, Buzby JS, et al. Immune thrombocytopenic purpura (ITP) plasma and purified ITP monoclonal autoantibodies inhibit megakaryocytopoiesis in vitro. Blood. 2003;102:887-95.

46. McMillan R, Wang L, Tomer A, Nichol J, Pistillo J. Suppression of in vitro megakaryocyte production by antiplatelet autoantibodies from adult patients with chronic ITP. Blood. 2004;103: 1364-9.

47. Perdomo J, Yan F, Chong BH. A megakaryocyte with no platelets: anti-platelet antibodies, apoptosis, and platelet production. Platelets. 2013;24:98-106.

48. Yang L, Wang L, Zhao CH, Zhu XJ, Hou Y, Jun P, et al. Contributions of TRAIL-mediated megakaryocyte apoptosis to impaired megakaryocyte and platelet production in immune thrombocytopenia. Blood. 2010;116:4307-16.

49. Li S, Wang L, Zhao C, Li L, Peng J, Hou M. CD8 + T cells suppress autologous megakaryocyte apoptosis in idiopathic thrombocytopenic purpura. Br J Haematol. 2007;139:605-11.

50. Stoll D, Cines DB, Aster RH, Murphy S. Platelet kinetics in patients with idiopathic thrombocytopenic purpura and moderate thrombocytopenia. Blood. 1985;65:584-8.

51. Kurata Y, Hayashi S, Kiyoi T, Kosugi S, Kashiwagi H, Honda S, et al. Diagnostic value of tests for reticulated platelets, plasma glycocalicin, and thrombopoietin levels for discriminating between hyperdestructive and hypoplastic thrombocytopenia. Am J Clin Pathol. 2001;115:656-64.

52. Hayashi S, Nishiyama M, Suehisa E, Kashiwagi H, Kurata Y, Tomiyama Y. Comparison between two methods for the measurement reticulated platelet and their clinical significance-flow cytometry (FCM) method and IPF method Using automated hematology analyzer (XE-2000). Rinsho Byori. 2009;57:103944.

53. Kaushansky K. Thrombopoietin. N Engl J Med. 1998;339: 746-54.
54. Kuter DJ, Gernsheimer TB. Thrombopoietin and platelet production in chronic immune thrombocytopenia. Hematol Oncol Clin North Am. 2009;23:1193-211.

55. Porcelijn L, Folman CC, Bossers B, Huiskes E, Overbeeke MA, van der Schoot $\mathrm{CE}$, et al. The diagnostic value of thrombopoietin level measurements in thrombocytopenia. Thromb Haemost. 1998;79:1101-5.

56. Stasi R, Sarpatwari A, Segal JB, Osborn J, Evangelista ML, Cooper $\mathrm{N}$, et al. Effects of eradication of Helicobacter pylori infection in patients with immune thrombocytopenic purpura: a systematic review. Blood. 2009;113:1231-40.

57. Kojouri K, Vesely SK, Terrell DR, George JN. Splenectomy for adult patients with idiopathic thrombocytopenic purpura: a systematic review to assess long-term platelet count responses, prediction of response, and surgical complications. Blood. 2004; 104:2623-34.

58. Li J, Yang C, Xia Y, Bertino A, Glaspy J, Roberts M, et al. Thrombocytopenia caused by the development of antibodies to thrombopoietin. Blood. 2001;98:3241-8.

59. Kuter DJ. New thrombopoietic growth factors. Blood. 2007;109: 4607-16.

60. Tomiyama Y. Thrombopoietin receptor agonists. Current \& emerging treatments for Immune Thrombocytopenia. (Salama A edit) Future Medicine, UK, 2013, pp 88-106.

61. Erickson-Miller CL, DeLorme E, Tian SS, Hopson CB, Stark K, Giampa L, et al. Discovery and characterization of a selective, nonpeptidyl thrombopoietin receptor agonist. Exp Hematol. 2005;33:85-93.

62. Cheng G, Saleh MN, Marcher C, Vasey S, Mayer B, Aivado M, et al. Eltrombopag for management of chronic immune thrombocytopenia (RAISE): a 6-month, randomised, phase 3 study. Lancet. 2011;377:393-402.

63. Saleh MN, Bussel JB, Cheng G, Meyer O, Bailey CK, Arning M, et al. Safety and efficacy of eltrombopag for treatment of chronic immune thrombocytopenia (ITP): results of the long-term, openlabel EXTEND study. Blood. 2013;121:537-45.

64. Shida Y, Takahashi N, Nohda S, Hirama T. Pharmacokinetics and pharmacodynamics of eltrombopag in healthy Japanese males. Jpn J Clin Pharmacol Ther. 2011;42:11-20.

65. Gibiansky E, Zhang J, Williams D, Wang Z, Ouellet D. Population pharmacokinetics of eltrombopag in healthy subjects and patients with chronic idiopathic thrombocytopenic purpura. J Clin Pharmacol. 2011;51:842-56.

66. Tomiyama Y, Miyakawa Y, Okamoto S, Katsutani S, Kimura A, Okoshi Y, et al. A lower starting dose of eltrombopag is efficacious in Japanese patients with previously treated chronic immune thrombocytopenia. J Thromb Haemost. 2012;10: 799-806

67. Kuter DJ, Bussel JB, Lyons RM, Pullarkat V, Gernsheimer TB, Senecal FM, et al. Efficacy of romiplostim in patients with chronic immune thrombocytopenic purpura: a double-blind randomised controlled trial. Lancet. 2008;371:395-403.

68. Kuter DJ, Rummel M, Boccia R, Macik BG, Pabinger I, Selleslag $\mathrm{D}$, et al. Romiplostim or standard of care in patients with immune thrombocytopenia. N Engl J Med. 2010;363:1889-99.

69. McMillan R, Bussel JB, George JN, Lalla D, Nichol JL. Selfreported health-related quality of life in adults with chronic immune thrombocytopenic purpura. Am J Hematol. 2008;83: $150-4$.

70. Shirasugi Y, Ando K, Miyazaki K, Tomiyama Y, Iwato K, Okamoto $\mathrm{S}$, et al. An open-label extension study evaluating the safety and efficacy of romiplostim for up to 3.5 years in thrombocytopenic Japanese patients with immune thrombocytopenic purpura (ITP). Int J Hematol. 2012;95:652-9. 
71. Reff ME, Carner K, Chambers KS, Chinn PC, Leonard JE, Raab $\mathrm{R}$, et al. Depletion of B cells in vivo by a chimeric mouse human monoclonal antibody to CD20. Blood. 1994;83:435-45.

72. Kimby E. Tolerability and safety of rituximab (MabThera). Cancer Treat Rev. 2005;31:456-73.

73. Cvetkovic RS, Perry CM. Rituximab: a review of its use in nonHodgkin's lymphoma and chronic lymphocytic leukaemia. Drugs. 2006;66:791-820.

74. Garvey B. Rituximab in the treatment of autoimmune haematological disorders. Br J Haematol. 2008;141:149-69.

75. Arnold DM, Dentali F, Crowther MA, Meyer RM, Cook RJ, Sigouin C, et al. Systematic review: efficacy and safety of rituximab for adults with idiopathic thrombocytopenic purpura. Ann Intern Med. 2007;146:25-33.

76. Patel VL, Mahévas M, Lee SY, Stasi R, Cunningham-Rundles S, Godeau B, et al. Outcomes 5 years after response to rituximab therapy in children and adults with immune thrombocytopenia. Blood. 2012;119:5989-95.

77. Provan D, Butler T, Evangelista ML, Amadori S, Newland AC, Stasi R. Activity and safety profile of low-dose rituximab for the treatment of autoimmune cytopenias in adults. Haematologica. 2007;92:1695-8.
78. Zaja F, Vianelli N, Volpetti S, Battista ML, Defina M, Palmieri S, et al. Low-dose rituximab in adult patients with primary immune thrombocytopenia. Eur J Haematol. 2010;85:329-34.

79. Hasan A, Michel M, Patel V, Stasi R, Cunningham-Rundles S, Leonard JP, et al. Repeated courses of rituximab in chronic ITP: three different regimens. Am J Hematol. 2009;84:661-5.

80. Neunert C, Lim W, Crowther M, Cohen A, Solberg L Jr, Crowther MA, American Society of Hematology. The American Society of Hematology 2011 evidence-based practical guideline for immune thrombocytopenia. Blood. 2011;117:4190-207.

81. Stasi R, Stipa E, Forte V, Meo P, Amadori S. Variable patterns of response to rituximab treatment in adults with chronic idiopathic thrombocytopenic purpura. Blood. 2002;99:3872-3.

82. Taylor RP, Lindorfer MA. Drug insight: the mechanism of action of rituximab in autoimmune disease-the immune complex decoy hypothesis. Nat Clin Pract Rheumatol. 2007;3:86-95.

83. Audia S, Samson M, Guy J, Janikashvili N, Fraszczak J, Trad M, et al. Immunologic effects of rituximab on the human spleen in immune thrombocytopenia. Blood. 2011;118:4394-400. 